\title{
El dilema de Otanes en América Latina ${ }^{1}$
}

The Otanes dilemma in Latin America

\section{Mario Gustavo Berrios Espezúa}

Universidad Católica de Santa María. Arequipa Perú.

\section{INFORMACIÓN}

\section{Historia del Artículo \\ Recepción: 08/06/2019 \\ Revisión: 05/08/2019 \\ Aceptación: 17/09/2019}

\section{Palabras Clave}

Democracia, gobierno, América

Latina.

\section{Key Words}

Democracy, government, Latin America.

\section{DOI}

https://doi.org/10.35286/veritas. v20i1.223

\section{RESUMEN}

El destacado politólogo italiano Norberto Bobbio (1909-2004) escribió un libro muy interesante acerca de las formas de gobierno en la historia del pensamiento político (Bobbio, 1992), el mismo que empieza con una "célebre discusión" entre tres personajes persas: Otanes, Megabyzo y Darío, acerca de cuál debería de ser la mejor forma de gobierno a instaurarse en Persia. Otanes es el defensor del gobierno de muchos (democracia), Megabyzo defiende el gobierno de pocos (aristocracia) y Darío apuesta por el gobierno de uno (monarquía), la democracia de Otanes es la única forma de gobierno que garantiza la igualdad ante la ley. Durante el estudio de las formas de gobierno (desde Platón hasta Marx), Bobbio llega a la conclusión de que la mejor forma de gobierno de todas es la democracia; sin embargo, en América Latina, la democracia ha asumido diversos tonos e incluso algunas formas de gobierno nada democráticos se hacen pasar como democráticos. En este trabajo desarrollaremos algunos puntos referidos a la célebre discusión que reseña Bobbio y su importancia en el actual debate acerca de la mejor forma de gobierno en América Latina; en segundo lugar, presentaremos el contexto neoliberal en el que la democracia en América Latina se instaura a inicios del siglo XXI; seguidamente, comentaremos algunos datos sobre la democracia en tres países de la región: Venezuela, Ecuador y Perú.

\begin{abstract}
The prominent Italian political scientist Norberto Bobbio (1909-2004) wrote a very interesting book about the forms of government in the history of political thought (Bobbio, 1992), in that book he begins with a "famous discussion" between three Persian characters: Otanes, Megabyzo and Dario, about what should be the best form of government to be established in Persia. Otanes is the defender of the government of many (democracy), Megabyzo defends the government of a few (aristocracy) and Dario defends the government of one (monarchy), Otanes democracy is the only form of government that guarantees equality before the law. During the study of forms of government (from Plato to Marx), Bobbio comes to the conclusion that the best form of government of all is democracy; however, in Latin America, democracy has assumed different tones and even some non-democratic forms of government pass themselves off as democratic. In this paper we will develop some points referring to the famous discussion that Bobbio reviews and its importance in the current debate about the best form of government in Latin America; second, we will present the neoliberal context in which democracy in Latin America was established at the beginning of the 21 st century; next, we will comment on some data about democracy in three countries of the region: Venezuela, Ecuador and Peru.
\end{abstract}

\section{INTRODUCCIÓN}

La célebre discusión, ¿Muchos, pocos o uno?

El libro La teoría de las formas de gobierno en la historia del pensamiento político de Norberto Bobbio (1992) se ha convertido en un clásico para quienes desean conocer un poco el proceso de construcción histórica de los principales conceptos y debates teóricos en torno a la ciencia política. Uno de estos debates es la ya también clásica discusión acerca de la mejor forma de gobierno para la sociedad ${ }^{3}$. El primer capítulo del libro de Bobbio contiene una cita extensa, pero muy importante, acerca de este debate clásico. La amplitud en la cita va de la mano con la importancia de la misma:

“...Otanes propuso entregar el poder al pueblo persa y argumentó su propuesta de la siguiente manera: "Me parece que ninguno de nosotros debe ser hecho monarca: sería

Correspondencia:

Mario Gustavo Berrios Espezúa ${ }^{2}$

mberrios@ucsm.edu.pe una cosa desagradable e injusta; pues visteis la insolencia de Cambises hasta donde se levantó y también habéis participado de la insolencia del Mago. Pero ¿cómo podría ser cosa ordenada un gobierno monárquico, si al monarca le está permitido hacer lo que quiera sin responder ante nadie? La monarquía haría salir incluso al mejor hombre de su norma natural cuando tuviese tal poder. La posesión de grandes riquezas genera en él la prepotencia, y desde el inicio la envidia le es connatural; teniendo esas dos cosas tiene toda la maldad: en efecto realiza las acciones más reprobables, unas dictadas por la prepotencia, otras por la envidia. Parecería razonable que el monarca-tirano fuese un hombre sin envidia, desde el momento que lo tiene todo. Pero se ha hecho al contrario de esto para con los súbditos, pues odia a los pocos buenos que han quedado, se complace con los peores, presta gran atención a las calumnias. Y lo más absurdo de todo: si en efecto lo admiras mesuradamente, está apesadumbrado, porque no es muy bien honrado, y si alguno lo honra mucho, está molesto como con un adulador. Pero ahora diré lo que es más grave: trastorna las leyes patrias, viola mujeres y mata a capricho. En cambio, el gobierno del pueblo lleva en primer lugar el más bello de los nombres, 
Isonomía (igualdad de derechos políticos); y en segundo lugar, nada hace de aquellas cosas que un monarca hace. Pues por sorteo se ejercen los cargos públicos, los magistrados son obligados a rendir cuentas del ejercicio del poder, toda decisión es sometida al voto popular: propongo pues, que nosotros rechacemos la monarquía para dar el poder al pueblo: pues todo es posible para el mayor número".

Pero Megabyzo exhortaba a entregarse a la oligarquía, diciendo esto: Lo que Otanes dijo para abolir la monarquía, eso también queda dicho por mí; pero lo que aconsejaba: conferir el poder al pueblo se ha apartado de la mejor opinión, pues nada hay más obtuso y prepotente que una multitud inepta. Y ciertamente, de ninguna manera es aceptable que unos hombres, huyendo de la insolencia de un tirano, caigan en la insolencia de un irresponsable populacho. Pues si aquel hace algo, lo hace dándose cuenta; pero a éste ni siquiera le es posible darse cuenta. Pues ¿cómo podría darse cuenta si no ha sido instruido, ni ha visto ningún bien, y se precipita, lanzándose sin inteligencia sobre los acontecimientos, semejante a un tormentoso río? Válganse de la democracia aquellos que piensan hacer daño a los persas; pero nosotros, habiendo elegido a un grupo de los mejores hombres, investíamos a estos con el poder, ya que en ellos estaremos nosotros mismos y es natural que de los mejores hombres sean las mejores decisiones.

Y Darío exponía su opinión diciendo: "Lo que dijo Megabyzo acerca del gobierno popular me parece haberlo dicho correctamente, no así lo concerniente a la oligarquía. Pues propuestas tres cosas y siendo todas muy buenas en principio, es decir, la mejor democracia, la mejor oligarquía y la mejor monarquía, afirmo que ésta es muy superior. Pues nada mejor podría aparecer que un solo hombre, el mejor, ya que, utilizando tal criterio, administraría intachablemente al pueblo; así también se mantendrían más en silencio los planes políticos contra los hombres malévolos. En una oligarquía, entre quienes practican la virtud para el bien público es fácil que nazcan graves enemistades personales: cada uno de aquellos quiere ser el jefe y hacer prevalecer su opinión por eso ellos llegan a odiarse recíprocamente; de aquí brotan las facciones, y de las facciones el delito. Del delito se llega a la monarquía, que por ello se muestra como el mejor gobierno. Y a la vez, cuando el pueblo gobierna, es imposible que no se origine la corrupción en la esfera pública; la corrupción no genera enemistades, sino sólidas amistades entre los malvados; los que actúan en contra del bien común lo hacen conspirando; y tal cosa existe hasta que alguno, habiéndose puesto al frente del pueblo, calma a los tales: $\mathrm{Y}$ de entre ellos éste, es entonces admirado por el pueblo; y siendo admirado, aparece siendo monarca. Y también por eso es claro que la monarquía es la mejor forma de gobierno. Y para decirlo en pocas palabras: ¿De dónde ha habido libertad para nosotros y habiéndola otorgado quién? ¿Acaso de parte del pueblo o de la oligarquía o del monarca? Tengo, por tanto, la opinión de que nosotros, habiendo sido liberados por un solo hombre, debemos mantener el régimen monárquico y conservar nuestras buenas instituciones patrias, pues no hay cosa mejor (Bobbio, 1992, págs. 15-17).

Considero que esta gran descripción no necesita mayores interpretaciones; lo que deseamos rescatar es el hecho que los personajes en cuestión no solo se dedican a defender la forma de gobierno que cada uno de ellos considera importante; sino que la mayor parte de sus argumentaciones se orientan a la crítica y desprecio a las otras formas que consideran negativas. Bobbio sintetiza el debate al afirmar que en éste podemos encontrar seis formas de gobierno usando dos criterios que se cruzan: ¿quién gobierna? Y ¿cómo gobierna? Tomando en cuenta esas distinciones, George Sabine (1945) hace una síntesis muy importante:

- Monarquía, un solo gobernante que ejerce el poder de acuerdo a la ley. Cuando deja de actuar de acuerdo a la ley, el gobierno se corrompe y por el abuso del poder se convierte en tiranía.

- Tiranía, el grupo de los notables se revelan y se pasa a la aristocracia.

- Aristocracia: gobierno reducido de pocos nobles que actúa de acuerdo a la ley. Cuando se corrompe y actúa en contra de la ley se pasa a la oligarquía. El pasaje de la aristocracia a la oligarquía es la rebelión.

- Oligarquía: siendo pocos viven bien y cuando los muchos pobres se rebelan derrocan a la oligarquía y se pasa a la democracia.

- Democracia: gobierno de muchos de acuerdo a la ley. Cuando se corrompe, se convierte en la oclocracia ${ }^{4}$.

- En este nivel es necesario hacer unas precisiones más acerca de las ventajas que Otanes plantea al gobierno democrático:

- "Isonomía", igualad de derechos políticos. Si extendemos la idea a derechos sociales, económicos, civiles, etc., estamos hablando de una de las características más importantes de la democracia y de la ciudadanía moderna: estatus de derechos y deberes.

- "Por sorteo se ejercen los cargos públicos". Esto lo entendemos como otro principio básico de la democracia y uno de los derechos fundamentales de todos los ciudadanos, me refiero a la igualdad ante la ley. Cualquier persona, que cumpla con los requisitos establecidos en el ordenamiento jurídico respectivo, puede acceder a cualquier cargo público. Esta igualdad de condiciones es lo que Giovanni Sartori llama la democracia social (Sartori, 2007)

- "Los magistrados son obligados a rendir cuentas del ejercicio del poder". Otro de los elementos fundamentales de la democracia moderna es la rendición de cuentas. Ésta se puede entender desde dos ópticas: la que se hace cada vez que los gobernantes acuden al parlamento a explicar la marcha del país; y, la que se hace cada vez que los

${ }^{1}$ Trabajo preparado para su presentación en el $9^{\circ}$ Congreso Latinoamericano de Ciencia Política, organizado por la Asociación Latinoamericana de Ciencia Política (ALACIP). Montevideo, 26 al 28 de julio de 2017.

${ }^{2}$ Correspondencia: mberrios@ucsm.edu.pe

${ }^{3}$ Es importante precisar que la reseña que hace Bobbio corresponde al gran historiador Herodoto.

${ }^{4}$ El término oclocracia hace referencia al gobierno de la muchedumbre o como sustenta Megabyzo "una multitud inepta" (Bobbio, 1992, pág. 16). 
gobernantes son llamados a explicar las decisiones que se toman. En las democracias modernas esta última forma de rendición de cuentas se hace ante el parlamento que es el primer poder del Estado y en el que se encuentra representada la nación.

- "Toda decisión es sometida al voto popular". La esencia misma de la democracia radica en la decisión de la mayoría, esta característica, en palabras de Bobbio evitaría la tiranía, la corrupción y otros males de los Estados actuales. Parafraseando a Ellen Meiksins Wood, el logro de la democracia formal y el sufragio universal representaron sin suda un inmenso avance histórico para la concepción moderna de democracia (Meiksins, 2000).

\section{El contexto actual: Capitalismo neoliberal, estado y} democracia en América Latina

Una característica que debemos resaltar ahora es el carácter mundial del desarrollo capitalista actual. Como bien señala Jaime Osorio el capitalismo es la primera organización económica y social que presenta una vocación mundial (Osorio, 2004). Si bien es cierto el desarrollo capitalista ha pasado por diversas etapas, es la del capitalismo neoliberal globalizado la etapa actual. En ese sentido, capitalismo neoliberal y globalización son dos elementos que caracterizan no solo la economía contemporánea, sino también la política. Es innegable entonces que los cambios económicos en la reproducción monopólica y financiera tienen consecuencias políticas de significación, particularmente en el campo estatal (Osorio, 2004)

No solo se han comercializado materias primas y productos manufacturados a escala global; también se han exportado e importado ideologías, formas de pensamiento y formas de gobierno. Por ejemplo, José María Vidal Villa sostiene que el principal obstáculo que se opone a la mundialización económica en nuestros días es la pervivencia de los Estados "nacionales" y que dificultan la homogeneización mundial (Vidal, 1996). Es innegable que a nivel económico, en la mayor parte de casos, la expansión comercial se ha facilitado tremendamente, en el ámbito político la homogeneización de formas de gobierno aún tiene ciertas resistencias a nivel global. Muchos países que han sido ocupados por la democracia norteamericana distan mucho de ser democracias en las que se respeten los derechos fundamentales y la igualdad y justicia primen en la población.

La imposición de formas específicas de gobierno, a la fuerza, ha demostrado ser un mecanismo equivocado si no se consideran los elementos sociales y culturales de dichas poblaciones.

Osorio puntualiza:

"En el capitalismo, como sistema mundial, siempre ha existido un ejercicio desigual de la soberanía, siendo mayor en las naciones imperialistas o centrales y menor en las naciones dependientes o periféricas. Lo que realmente se pone en cuestión es la centralidad del Estado en materia de poder político, ante el surgimiento de nuevos centros de decisión. Se destaca la enorme injerencia ganada por organismos financieros internacionales. El Estado se nos presenta en este cuadro como una entidad frágil y débil ante procesos y nuevos actores que lo rebasan y que limitan su soberanía" (Osorio, 2004).

No solo el Estado se vuelve frágil y débil, la democracia como la forma de gobierno implementada en la mayor parte de naciones occidentales se ha debilitado, en muchas de las naciones latinoamericanas se encuentra en estado de emergencia. No ha sabido responder a las grandes expectativas que ella ha generado; muy por el contrario, ha defraudado sus propios principios. Sería mejor decir que los políticos que ejercen su accionar "guiados" por los principios de la democracia han defraudado a la ciudadanía. A nivel global la crisis del sistema democrático neoliberal parece haber encontrado su punto más álgido con la elección de Donald Trump en los Estados Unidos, la denominada "democracia perfecta". Al parecer la democracia norteamericana se ha convertido en la oclocracia que temían los antiguos filósofos griegos.

El caso Latinoamericano merece una consideración especial como ejemplo de la imposición ideológica y política de los países centrales.

El modelo de desarrollo implementado por la Comisión Económica para América Latina (CEPAL) de industrialización por sustitución de importaciones fue el inicio de una mayor intervención del Estado en aspecto económicos y de mercado asumiendo roles de interventor, promotor, planificador, etc., a efecto de contribuir de una manera mucho más activa en el desarrollo de la región.

Sin embargo, es necesario recordar que este modelo que auguraba el desarrollo e independencia económica de las naciones latinoamericanas de los Estados Unidos necesitaba de grandes cantidades de dinero para implementarse, recursos económicos con los que no se contaba. La pelota estuvo nuevamente en manos de los países desarrollados quienes vieron peligrar su influencia económica y política en dichas naciones si lograban la tan anhelada industrialización, motivo por el cual no apoyaron y, en algunos casos, boicotearon las acciones iniciadas por algunos gobiernos latinoamericanos conducentes al desarrollo.

Este modelo fue muy cuestionado por los neoliberales, encabezados por Ronald Reagan (EE. UU.) y Margaret Tatcher (Reino Unido), quienes eliminaron el Estado de bienestar en sus respectivos países e impulsaron una fuerte política internacional de implementación del neoliberalismo en los países en vías de desarrollo o del tercer mundo como eran conocidos en esos años.

En América Latina dicha implementación ortodoxa siguió algunos patrones, que Juan Rodríguez (2008) resume de la siguiente manera:

- En Chile (1973). Chile fue el laboratorio en donde se experimentó por vez primera el proyecto neoliberal. Fueron los economistas de la Universidad de Chicago, llamados "Chicago boys", bajo la dirección del premio Nobel de economía Milton Friedman, los encargados de conducir su implantación durante el régimen de Augusto Pinochet.

- En Bolivia (1985), se aplicó la política del "shock", bajo el gobierno de Víctor Paz Estenssoro, el mismo que implicaba la aplicación drástica, radical y con mayor celeridad posible del neoliberalismo.

- En México (1988) el presidente Carlos Salinas de Gortari fue condecorado por la primera ministra inglesa, Margaret Tatcher, por la prontitud, fidelidad y ortodoxia con que implementó el proyecto neoliberal.

- En Argentina (1989) se dio inicio a la era neoliberal con la implementación del "decálogo Menemsiano" en el gobierno del presidente Carlos Saúl Menem y su ministro 


\section{Berrios Espezúa / VÉRITAS Vol. 20 N¹ (2019) 37-44}

de economía Domingo Cavallo.

- En Venezuela (1989) la política neoliberal se inició bajo la administración del presidente Carlos Andrés Pérez, cuya consecuencia inmediata fue el "caracazo".

- En Brasil (1989) se inició con el presidente Fernando Collor de Melo y fue continuado por Fernando Henrique Cardoso.

- En Perú (1990) el neoliberalismo llegó a suelo peruano con el gobierno de Alberto Fujimori Fujimori, hoy preso, quien, habiendo ganado las elecciones con un programa heterodoxo y populista, contra el programa neoliberal del candidato arequipeño Mario Vargas Llosa, una vez asumido el poder aplicó el famoso "fujishock" con total radicalidad.

Es en base a la aplicación del neoliberalismo, en reemplazo del populismo, como nueva "matriz sociopolítica" (Garretón, 2004) que se dan una serie de transformaciones en varios aspectos de la realidad: el político, económico, social, cultural, etc.

Es también necesario puntualizar que para diversos autores el neoliberalismo implementado en América Latina ha resultado ser un rotundo fracaso. Actualmente las economías latinoamericacanas, luego de un periodo breve de bonanza económica parecen haberse estancado y el crecimiento económico ha empezado a retroceder. Hoy en día los gobiernos latinoamericanos son muy débiles y la democracia que los sostienen parece tambalear. Las sociedades latinoamericanas han entrado en una fuerte crisis social y moral de la cual la política neoliberal de máxima ganancia y lucro excesivo es la principal responsable. El individualismo y la competencia a toda costa y su implantación en el imaginario social de los latinoamericanos es la peor herencia de este sistema.

Como podemos ver las aristas son múltiples, como lo son los espacios en los cuales la ideología neoliberal ha intentado penetrar. Para objeto del presente trabajo, solo analizaremos las repercusiones políticas del neoliberalismo, específicamente en el tema de la democracia.

El término democracia es una expresión ampliamente extendida en el mundo teórico político, y en la práctica es una herramienta poderosa que permite la participación de la sociedad civil en el proceso de gobernar por el pueblo y para el pueblo; sin embargo, esta herramienta es aún muy frágil, en especial en los países en vías de desarrollo.

Los diversos gobernantes han asumido varias posiciones en torno a ella, unos fortaleciéndola y otros debilitando sus bases hasta destruirla. Por lo tanto, los primeros deben de, no solo continuar su arduo trabajo y recibir el apoyo de la ciudadanía, sino también de utilizar los recursos en pro de los mejores intereses de nuestra región.

La democracia es un sistema político, social y económico de hombres no sólo libres e iguales ante la ley, sino en las relaciones sociales en la vida cotidiana, y es esta libertad la que nos da a escoger entre participar o no participar en los asuntos públicos. Retomando algunas ideas desarrolladas anteriormente, podríamos afirmar, siguiendo la definición del cuento de Otanes, que la democracia debe sustentarse en los derechos y deberes, la igualdad, la rendición de cuentas y el respeto a las decisiones del pueblo.

Una sociedad verdaderamente democrática debe aspirar al desarrollo humano, lo que significa decir que todas las acciones políticas, económicas, culturales, educativas, etc., deben estar orientadas a lograr el desarrollo de la comunidad.

Lamentablemente en América Latina debemos preguntarnos si hay o no democracia, en una región con un sistema económico injusto, donde los Estados no garantizan la salud de la ciudadanía, donde impera la injusticia, donde la educación es para los que más dinero tienen, donde la seguridad está en peligro y donde la clase política está desprestigiada $^{5}$ al extremo que la ciudadanía no se siente identificada con sus representantes, los mismos que fueron elegidos de manera democrática.

Ahora bien, la respuesta a la pregunta anterior simplemente se la dejamos al lector, lo que si podemos mencionar es que la democracia moderna, no la clásica, es decir la occidental presenta ciertas características que la hacen especial y distinta a otros sistemas de gobierno en el mundo: Principio de soberanía popular, por medio del cual las instituciones estatales gubernamentales sólo pueden pretender reconocimiento popular cuando han sido elegidas por el pueblo. Principio de la igualdad de las personas, no queremos manifestar que todos somos iguales, ya que hacerlo sería negar la dinámica y diferencia social que hace a cada ser humano único, sino a la igualdad de derechos y deberes que todos poseemos, independientemente de nuestro sexo, género, creencia, raza, etc. Principio de libertad personal, todos somos libres de hacer lo que creamos conveniente hacer, pero esta libertad tiene un límite, podemos hacer lo que queramos, siempre y cuando no afectemos al prójimo, esa es la libertad fundamental en democracia. Y el principio de control del gobierno, a través del cual los ciudadanos hacemos efectivo nuestro papel en el proceso de participación política y ciudadana.

En este punto creemos importante mencionar algunos datos estadísticos que nos pueden mostrar la realidad y percepción de la democracia en América Latina; para ello hemos tomado los datos del Informe 2016 de Corporación Latinobarómetro (2016).

Respecto al apoyo a la democracia, en comparación con un régimen autoritario, la preferencia hacia la democracia ha ido decreciendo desde el el año 2010, en que el apoyo era del $61 \%$, pasando a un 56\% en el año 2013, hasta llegar a un $54 \%$ en el 2016. Si bien es cierto que los datos no muestran que el apoyo al autoritarismo haya aumentado (de hecho del 2010 al 2016 se mantiene en un $15 \%$ ), donde si se ha notado un incremento ha sido en la posición de muchos encuestados de mantenerse indiferentes frente a ambos regímenes de gobierno: en el 2010 el procentaje de población indiferente era del $16 \%$, subió al $20 \%$ en el 2013 y ahora se ubica en el $23 \%$. De estos datos podemos extraer algunas ideas importantes: en primer lugar, es falsa la percepción de

${ }^{5}$ En diciembre del 2016 el Departamento de Justicia de los Estados Unidos publicó una investigación sobre la constructora brasileña Odebrecht, en la que se detalla que la misma habría realizado coimas de dinero y sobornos a funcionarios públicos de 12 países, para obtener beneficios en contrataciones públicas. Los gobiernos latinoamericanos comprometidos, hasta el momento son: Brasil, Colombia, México, Panamá, Guatemala, República Dominicana, Perú, Argentina, Venezuela y Ecuador. 
que los latinoamericanos preferios "mano dura" (entiéndase, autoritarismo) en vez de democracia; en segundo lugar, existe una creciente indiferencia hacia la democracia como sistema de gobierno, ¿a qué podría deberse esta indiferencia? Siguiendo algunos planteamientos de Vallès y Ballart (2012), creemos que esta indiferencia es no solo hacia la democracia, sino hacia la política en general: los ciudadanos ven el mundo de la política en democracia como alejado de ellos, la adminsitración pública está llena de formalidades y solemnidades que poco o nada ayudan a solucionar los problemas cotidianos. Existe una percecpión de los políticos como animales que luchan por su supervivencia, mientras que la sociedad en su conjunto tiene que buscar sus propios medios de subsistencia.

Otro indicador importante recogido por el Informe de Latinobarómetro es el referido a la satisfacción con la democracia. Al igual que la variable anterior, la insatisfacción con la democracia ha llegado a niveles no antes medidos: pasamos de $52 \%$ de población insatisfecha con la democracia en el 2010 a un $62 \%$ en el 2016. Luego de cerca de 20 años de instauración de regímenes democráticos en casi toda América Latina, estas cifras lo que nos generan es preocupación, más aún si las relacionamos con los datos anteriores de apoyo a la democracia. Al parecer la percepción de los latinoamericanos es que la democracia no responde a sus necesidades, que los principios de igualdad, libertad, etc., son simples declaraciones para las tribunas o para las campañas electorales. No hay una respuesta efectiva ante las necesidades más urgentes de la población, estamos frente a gobiernos que cumplen con ser elegidos por voto popular, pero que una vez en el cargo olvidan (o les hacen olvidar) las promesas electorales. Los planes, programas, en fin, las políticas públicas no encuentran un reflejo adecuado en lo que la gente necesita, peor aún, no son tan efectivas como es el deseo de la población.

Realacionado a lo anterior, otra de las preguntas presentadas en el Informe de Latinobarómetro es ¿Para quién se gobierna? Es de suponer que se gobierna para el pueblo, quien a fin de cuentas coloca a los gobernantes en dicha posición; sin embargo los datos muestran todo lo contario. Mientras en el 2010 el $20 \%$ de los encuestados considera que se gobierna para todo el pueblo, ahora ese porcentaje solo es del $22 \%$. Por otro lado, en el 2010 el $64 \%$ de los encuestados consideraba que se gobernaba para los grupos o clases poderosos, al 2016, esta cifra se ha incrementado, llegando al $73 \%$ de personas encuestadas. Como bien lo señalan Vallès y Ballart: "La sensación de lejanía entre ciudadanía y políticos es un lugar común cuando se habla de los problemas actuales de los sistemas democráticos" (2012, pág. 29). A diferencia del siglo pasado, en que el desarrollo de los medios de comunicación era aún limitado, hoy en día contamos con medios no convencionales por los cuales nos enteramos de los "arreglos" o de los juegos de intereses al interior de la misma democracia. El internet y las redes sociales virtuales juegan un papel importantísimo para que la población no crea tan ingenuamente como antes lo que los medios oficiales informan. Si dejamos que la democracia siga pareciendo un proyecto político que beneficie a unos pocos sin tomar en cuenta la voz y propuestas de las grandes mayoría, el panorama de insatisfacción seguirá creciendo.

Finalmente, es importante recordar que la democracia, como todo régimen de gobierno, se sustenta en sus instituciones, las mismas que deben contar con un apoyo adecuado de la ciudadanía. En ese sentido, los datos del Informe de Latinobarómetro respecto a confianza en instituciones son: confianza en las Fuerzas Armadas, 50\%; confianza en la Policía Nacional, 38\%; confianza en los órganos electorales, 32\%; confianza en el gobierno, $28 \%$; confianza en el poder judicial, 26\%; confianza en el congreso, $25 \%$; y , confianza en los partidos políticos, $17 \%$. Niguna de estas instituciones sobrepasa la mitad. No es de estrañarse que luego de años de dictaduras militares, sean las Fuerzas Armadas las que mantengan un alto nivel de confianza, recordemos que se autodenominan las fuerzas "tutelares" de la nación. Lo preocupante es que los tres poderes del estado (ejecutivo, legislativo y judicial) cuenten con tan poco nivel de confianza; si bien es cierto esta situación es alarmante, no es muy extraña para nuestro contienente, si tomamos como base las variables reseñadas anteriormente. Es importante mencionar que son los ciudadanos quienes desconfían de las personas que ellos mismos pusieron al frente de dichas instituciones, por lo que es importante fortalecer y en algunos países formar, una cultura ciudadana de compromiso con la democracia y la política, ser ciudadanos activos y no ciudadanos cada cinco años.

Como veremos más adelante, el dilema de Otanes surge cuando estas y otras características de la democracia, o poliarquía, como la llama Robert Dahl cambian o no todas se cumplen; entramos entonces al análisis de algunos casos paradigmáticos de "democracias" en América Latina, aquellas que Steven Levitsky y Lucan Way han denominado autoritarismos competitivos (Levitsky \& Way, 2004).

\section{Estudios de casos: VENEZUELA, ECUADOR Y PERÚ}

En base al contexto anterior intentaremos un breve análisis acerca del régimen político que impera hoy en día en tres países latinoamericanos: Venezuela, Ecuador y Perú. La elección, si bien es cierto en un primer momento al azar, ahora se sustenta en los datos del Informe de Latinobarómetro 2016, en los que estos tres países presentan una disminución significativa entre el 2015 y 2016 respecto al apoyo a la democracia (Perú y Ecuador) y Venezuela que presenta una disminución muy significativa de apoyo a la democracia. Regresaremos a esa información más adelante.

Venezuela y Ecuador, históricamente hablando tienen un pasado colonial similar, formaban lo que se denominaba la Gran Colombia (junto con Colombia) y fueron el centro de operaciones y mando de la Corriente Libertadora del Norte, encabezada por Simón Bolívar. Es bajo la figura de este libertador que se han erigido las dos grandes figuras que han gobernado estos países en los últimos años: Hugo Chávez en Venezuela y Rafael Correa en el Ecuador.

Existen algunas características en la forma de gobierno de ambos personajes que, siguiendo a Levitsky y Way (2004), nos hacen concluir que no estamos frente a ninguna democracia ni a ningún autoritarismo, estamos frente a un híbrido, una mezcla mal hecha de democracia y dictadura.

En primer lugar, las características democráticas que podríamos encontrar en la Venezuela de Chávez fueron la existencia de instituciones políticas formales y la convocatoria a elecciones generales, como único medio de llegar al poder. Por otro lado, las características autoritarias del régimen se pueden resumir en el abuso irrestricto, por parte del presidente, de la ley y del poder para obtener 
réditos personales y políticos: el control absoluto de los medios de comunicación masiva; el control de las principales instituciones electorales, judiciales e incluso legislativas; y la persecución y silenciamiento a la oposición política.

En el caso del Ecuador de Correa, democráticamente hablando, tenemos un régimen presidido por un civil, existen instituciones políticas formales, las elecciones son el único medio para llegar al poder y existen mecanismos de participación ciudadana de la oposición política. Sin embargo, autoritariamente el presidente abusó del poder que poseía para obtener sus propios beneficios políticos, hubo control de los medios de comunicación y existió competencia política, pero esta muchas veces no era justa ni equilibrada.

Existen algunos ejemplos de las características teóricas que resumimos en el párrafo anterior. En el caso ecuatoriano, el presidente Correa fue elegido presidente democráticamente, en elecciones generales del año 2006; sin embargo, una vez en el poder cambió las reglas electorales, de tal forma que se posibilitó su reelección en el 2009 y en el 2013. También es conocida la falta de tolerancia del presidente ecuatoriano hacia los medios de comunicación que están en contra de las acciones que toma, es famosa la demanda por una suma millonaria a un periodista por una información brindada acerca de Correa.

En el caso de Venezuela, la situación era aún más escandalosa. Si bien es cierto que Chávez no era un civil, asumió el mando presidencial por medio de elecciones democráticas en 1999; sin embargo, a pesar de jurar por la constitución que no la cambiaría, éste hizo las modificaciones constitucionales necesarias para poder garantizar su permanencia por más años en el poder, ganando las elecciones del 2001, 2006 y 2013.

Es también ampliamente conocido el abuso que cometió de las leyes y del poder para sus beneficios y el de sus allegados, el control de los medios de comunicación y la expropiación de aquellos propietarios de medios que el presidente consideraba "enemigos de la revolución bolivariana" y "lacayos del Imperio (EE. UU.)". Como bien sabemos, Chávez Frías falleció en 2013; pero el aparato de autoritarismo competitivo que forjó y dejó en herencia a Venezuela se mantiene, igual de fuerte. El ejemplo más claro fue el proceso electoral en que fue elegido Nicolás Maduro y que estuvo marcado por un irrestricto control del ejecutivo hacia los órganos electorales. Hoy en día la situación política en Venezuela representa un gran reto para la clase política, ya que sus efectos se vienen viendo en lo social y económico.

En lo referente al caso peruano, si bien es cierto se cuenta con una institucionalidad democrática relativamente consolidada, se hace evidente en los últimos años que la sociedad civil organizada clama por una ampliación de la democracia que vaya más allá de lo meramente formal y transite de la democracia representativa hacia la democracia participativa; o como plantea Walter Varillas, "la democracia participativa como complemento de la democracia representativa" (Varillas, 2005: 24).

En el Perú impera un régimen democrático, en el sentido tradicional, pero que está acomodado al gusto del capitalismo norteamericano, como Colombia y México; nuestros gobernantes democráticamente elegidos (desde Alberto Fujimori hasta Ollanta Humala) han ganado las elecciones con un plan de gobierno diametralmente opuesto a lo que hicieron una vez en el poder; sus plataformas de lucha y planes de gobierno fueron los típicos de la centro izquierda o populistas; sin embargo, una vez en el poder, terminaron gobernando con el plan de gobierno del contrincante vencido, el mismo que impulsaba mayor neoliberalismo político y económico. Este fenómeno tan particular de ganar y gobernar es lo que Yusuke Murakami denomina "política de entrar por la izquierda y salir por la derecha" y que se encuentra ampliamente documentado en su obra La actualidad política de los países andinos centrales en el gobierno de izquierda (Murakami, 2014).

Veamos algunos datos comparativos del apoyo a la democracia en estos tres países, del 2010 al 2016:
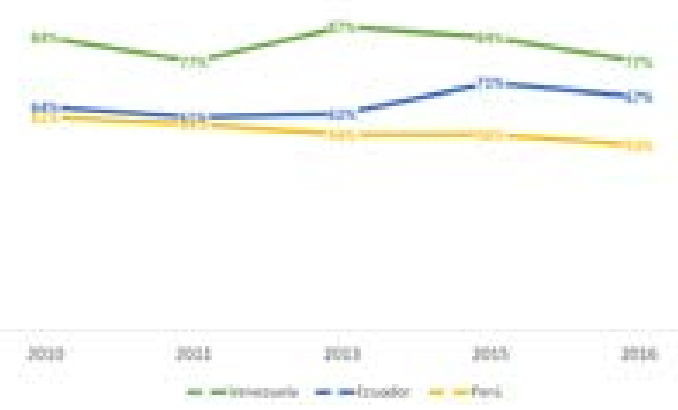

Fig. 1: Apoyo a la democracia en Venezuela, Ecuador y Perú, 2010-2016. Fuente: Informe de Latinobarómetro 2016.

La información que podemos apreciar en la Ilustración 1 nos resume a grandes rasgos la situación en general de apoyo a la democracia en los tres países de estudio. Como lo señalábamos líneas arriba, en Ecuador y Perú podemos observar una significativa reducción en el apoyo a la democracia, con -4 puntos porcentuales en el Ecuador y -3 puntos porcentuales en el Perú solo en los últimos dos años. En el caso de Venezuela podemos apreciar una muy significativa reducción en el apoyo a la democracia del 2015 al 2016: -7 puntos porcentuales. En este último caso creemos que la situación política, social y económica crítica que vive el país ha llevado a tan gran disminución en la percepción ciudadana y el consiguiente apoyo a este régimen de gobierno.

En líneas anteriores hablábamos de la libertad individual como uno de los derechos políticos más importantes que debiera garantizar la democracia, veamos algunos datos:

Tabla 1: Orden versus libertad individual en Venezuela, Ecuador y Perú, 2010-2016. Fuente: Informe de Latinobarómetro 2016.

\begin{tabular}{ccc}
\hline PAÍSES & $\begin{array}{c}\text { ORDEN SOBRE } \\
\text { LIBERTAD }\end{array}$ & $\begin{array}{c}\text { LIBERTAD SOBRE } \\
\text { ORDEN }\end{array}$ \\
\hline Venezuela & $42 \%$ & $56 \%$ \\
Ecuador & $47 \%$ & $49 \%$ \\
Perú & $56 \%$ & $37 \%$ \\
\hline
\end{tabular}

Antes del análisis de cada caso es importante mencionar los datos globales, a nivel de toda América Latina. La preeminencia del orden sobre la libertad tiene una aceptación del $52 \%$ en los ciudadanos latinoamericanos, mientras la preeminencia de la libertad individual sobre el orden solo 
llega a un $44 \%$. Al realizar el análisis en cada país podemos identificar que en Venezuela se valora más la libertad que el orden, en Ecuador la situación es casi pareja en referencia a ambos elementos y en el Perú tenemos una mayor valoración del orden por encima de las libertades individuales, siguiendo la tendencia de toda América Latina. En el caso de Venezuela parece claro el hecho que desde que asumió el gobierno Chávez Frías éste ha tratado de imponer el orden a toda costa (como buen militar). Sin embargo los últimos años y, en especial, desde que Maduro llegó al poder, parece que los venezolanos han dado una mayor importancia a las libertades, muchas de ellas recortadas por el régimen actual. En los casos venezolano y ecuatoriano habría que preguntarnos qué tipo de libertad es la que valoran sus ciudadanos. "La liberal, que sostiene que ser libre significa no estar sometido a interferencias; la republicana, que afirma que ser libre quiere decir no depender de la voluntad arbitraria de otros individuos; y la democrática, que defiende que ser libre significa, ante todo, poder decidir las normas que regula la vida social" (Bobbio y Viroli, 2002, págs. 33-34). Por otro lado, en el caso peruano se presenta como el típico país positivista Comteano en el que el orden está por encima de todo: "El orden como base, el progreso como fin" destacaba Auguste Comte, padre de la sociología. Al parecer los últimos años de "bonanza" economíca han hecho creer a los peruanos que las cosas tal como están, garantizan el crecimiento y que poco o nada se debe hacer para cambiar el statu quo. Una de las libertades más importantes en democracia es la libertad de información, en ese sentido los datos muestran que a nivel de América Latina hay muy poca aceptación al hecho de que el presidente controle los medios de comunicación, solo el $30 \%$. En los tres países de estudio la tendencia es similar: Venezuela, 29\%; Ecuador, 39\%; y Perú, 26\%.

Otro de los fundamentos clásicos de la democracia es el referido a su utilidad, ¿sirve realmente la democracia para solucionar los problemas de la población? Según los datos del Informe de Latinobarómetro 2016, el 61\% de los ciudadanos de latinoamérica manifiesta una respuesta afirmativa ante dicha pregunta. En nuestros países de estudio la tendencia parece ser la misma: en Venezuela es un $72 \%$, en Ecuador, 64\% y en Perú 52\%. En este nivel de análisis habría que considerar varios factores que explican estos datos. Uno de ellos es la figura del líder. La presencia de líderes carismáticos (Ecuador) y tradicionales (Venezuela) generan una imagen de que lo que ellos dicen representar está bien y funciona bien. Sumado a ello, otro factor importante es el control que se hace de los medios de comunicación y difusión de la información, que muchas veces son usados como canales de propaganda política para mostrar lo que el líder quiere hacer creer a la gente que pasa, ocultando la realidad. Finalmente, sin acercarme a terminar el debate, creo que la efectividad de políticas públicas demagógicas hacen que el sistema democrático sea visto como eficaz en el corto plazo. En el caso del Perú, no existe un liderazgo claro, ni control de medios de comunicación, ni política demagógica que cree la ilusión de que el sistema democrático solucione los problemas de la gente, de ahí el gran descontento. Un dato que confirma dicha afirmación se encuentra en el mismo Informe: el 39\% de peruanos considera que no importa un gobierno no democrático si resuelve los problemas de la gente; frente a un $31 \%$ de venezolanos que piensa lo mismo.

Finalmente, puntualizamos que la democracia tiene que ver mucho con la rendición de cuentas; eso pasa necesariamente por el control y la libertad de crítica que debe tener la ciudadanía frente a los distintos actos que realiza el gobierno. Un dato importante es la percepción acerca de la libertad de crítica que existe. El 51\% de latinoamericanos considera que si existe libertad de crítica. En Venezuela la cifra es igual, en Ecuador llega a un $34 \%$ y en el Perú se dispara a un 53\%. Podemos ensayar algunas hipótesis que expliquen estos datos, como por ejemplo el miedo. Conocedores del poco nivel de tolerancia a la oposición en Venezuela, cualquiera podría cuestionar la cifra de que más de mitad de ciudadanos cree que hay libertad de crítica. Tal vez la explicación vaya más por lo subjetivo: el miedo a las represalias por parte del régimen. Otro elemento puede ser la intervención en los mecanismos de control, lo que genera una ilusión de vigilancia. El control deja de ser efectivo cuando se asume como una formalidad más que poco o nada influye en las decisiones de los gobernantes, en ese marco la construcción y fortalecimiento de la confianza juega un papel fundamental. Solo para citar unos datos finales, la confianza interpersonal en América Latina es de 17\%. En Venezuela solo el $14 \%$ de ciudadanos confía entre ellos, en Ecuador la cifra llega al $21 \%$ y en Perú a $15 \%$. Como herencia de un pasado reciente autoritario, tenemos que a nivel de América Latina, las Fuerzas Armadas son la institución en la que más se confía, con un $51 \%$ de confianza. En el otro extremo encontramos a los partidos políticos con solo un $17 \%$ de confianza ciudadana. Estas cifras debieran llamarnos la atención hacia dónde vamos con nuestra democracia y qué debemos cambiar en este régimen.

\section{A manera de cierre}

Creemos que el dilema acerca de la vigencia y relativa eficiencia de la democracia, sigue tan vigente como lo fue en la época de los filósofos griegos; en ese sentido sigue siendo un tema clásico en ciencia política que a pesar del tiempo nos sigue planteando preguntas y generando retos no solo desde el mundo académico, sino en la práctica misma de la política.

Las condiciones sociales, económicas y políticas del mundo contemporáneo nos reafirman en la idea de repensar el sistema democrático a la luz de la fuerte crítica que sobre él existe hoy en día. Diversos movimientos sociales manifiestan su descontento con la democracia occidental porque, en pocas palabras, no ha sabido responder a las necesidades de la sociedad.

Pero esto puede y, de hecho, debe cambiar. Para ello es necesario crear una cultura de participación y control ciudadano efectivo; así como también un fortalecimiento de los partidos políticos que tenemos para una adecuada selección de cuadros políticos que nos representen en el futuro. Con todos sus beneficios y desventajas creemos que la apuesta por una democracia realmente participativa y representativa debe ser el sendero por el cual nuestros pueblos deben seguir. 


\section{REFERENCIAS BIBLIOGRÁFICAS}

1. Altamirano, T. (2007). Transnacionalismo y movilidad del capital humano. En A. Panfichi, Aula Magna. Migraciones internacionales (págs. 39-65). Lima: PUCP.

2. Bobbio, N. (1992). La teoría de las formas de gobierno en la historia del pensamiento político. México: Fondo de Cultura Económica.

3. Bobbio, N., \& Viroli, M. (2002). Diálogo en torno a la república. Barcelona: Tusquets Editores.

4. Corporación Latinobarómetro. (2016). Informe 2016. Buenos Aires.

5. Corvetto, P. (2013). Maestría en Ciencia Política y Gobierno. Curso 3: Procesos Políticos en el Perú y América siglo XX. Lima|: PUCP.

6. Garretón, M., Cavarozzi, M., Cleaves, P., Gereffi, G., \& Hartlyn, J. (2004). América Latina en el siglo XXI. Hacia una nueva matriz sociopolítica. Santiago de Chile: LOM Ediciones.

7. Hillmann, K.-H. (2005). Diccionario Enciclopédico de Sociología. Barcelona: Herder.

8. Laclau, E. (2005). La razón populista. Buenos Aires: Fondo de Cultura Económica.

9. Levitsky, S., \& Way, L. (2004). Elecciones si democracia. El surgimiento del autoritarismo competitivo. Estudios Políticos(24), 159-176.

10. Mari, E. (2000). El ciclo de la Tierra. Minerales, materiales, reciclado, contaminación ambiental. Buenos Aires: FCE.

11. Meiksins, E. (2000). Democracia contra capitalismo. México: Siglo XXI.

12. Murakami, Y. (2014). La actualidad política de los países andinos centrales en el gobierno de izquierda. Lima: CIAS, Center for Integrated Area Studies, Kyoto University, IEP.

13. Osorio, J. (2004). El Estado en el centro de la mundialización: la sociedad civil y el asunto del poder. México: Fondo de Cultura Económica.

14. Pease, H. (2013). La Política en el Perú del siglo XX. Lima: PUCP.

15. Rodríguez, J. (2008). Globalización, neoliberalismo y universidad: pautas para una reforma universitaria. Arequipa: UNSA.

16. Sabine, G. (1945). Historia de la teoría política. México: Fondo de Cultura Económica.

17. Sartori, G. (2007). ¿Qué es la democracia? Buenos Aires.: Taurus.

18. Singer, H. (Julio de 1989). El desarrollo en la posguerra. Lecciones de la experiencia de 1945 a 1985. Comercio Exterior, 39(7), 597-617.

19. Stiglitz, J. (2006). Cómo hacer que funcione la globalización. Madrid: Taurus.

20. Uribe, C. (2008). Un modelo para armar. Teoría y conceptos de desarrollo. Lima: Fondo editorial PUCP.

21. Vallès, J., \& Ballart, X. (2012). Política para apolíticos. Contra la dimisión de los ciudadanos. Barcelona: Editorial Planeta.

22. Varillas, W. (2005). Propuesta de sistema político local de participación y concertación. Lima: IDEAS.

23. Vidal, J. M. (1996). Mundializacion: diez tesis y otros artículos. Barcelona: Icaria. 